\title{
Changes in Perceived Work-from-Home Productivity during the Pandemic: Findings from Two Waves of a Covid-19 Mobility Survey
}

\author{
Xiao Shi $^{1}$ (D), Mary Richards ${ }^{2}$ (D), Anne Vernez Moudon ${ }^{1}$ (D), Brian H.Y. Lee ${ }^{2}$ (D), Qing Shen ${ }^{3}$ (D), Xuegang Ban ${ }^{4}$ (D) \\ ${ }^{1}$ Urban Form Lab, Department of Urban Design and Planning, University of Washington, ${ }^{2}$ Puget Sound Regional Council, ${ }^{3}$ Department of Urban Design and \\ Planning, University of Washington, ${ }^{4}$ Department of Civil \& Environmental Engineering, University of Washington \\ Keywords: work from home, teleworking, work productivity, commute trip reduction, transportation demand management, natural experiment, covid-19 \\ https://doi.org/10.32866/001c.32556
}

A two-wave survey of workers in Seattle revealed an increase in self-reported work productivity over time for those who shifted to work from home (WFH) since the outbreak of Covid-19. Teleworkers with higher household income adapted better and were more likely to report an increase in productivity as they continued WFH. While those living with friends and relatives were more likely to report a decrease in productivity as they telework for longer. Commute trip reduction programs might encourage the portion of the population with such characteristics to continue WFH after the pandemic subsides and provide support to those with fewer recourses to telework productively if they choose to.

\section{Questions}

The Covid-19 pandemic has impacted all aspects of life, most noticeably that of transitioning to work from home (WFH) for hundreds of millions of workers worldwide (Soares, Bonnet, and Berg 2021). By eliminating commute trips, WFH can contribute to transportation efficiency and environmental quality. Understanding the impact of the current mandated and prolonged WFH situation on work productivity (Kazekami 2020; Nakrošienè, Bučiūnienè, and Goštautaite 2019; Pigini and Staffolani 2019) might inform commute trip reduction policies after the pandemic subsides. Complementing previous research (Shi et al. 2020), this study explores the impact of WFH on teleworkers' productivity over time. We ask:

1. Did perceived work productivity change as workers continued to WFH over time?

2. Did the relationships between socioeconomic status (SES), lifestyle, and perceived teleworking productivity differ between spring and fall 2020 ?

\section{Methods}

\section{Study and Survey Design}

Two waves of a cross-sectional survey were conducted in the spring (AprilJune) and fall (October-November) of 2020. The data were pooled for analysis to identify differences over time, but the results do not infer causal relations. Consistent questions asked respondents about changes in their work productivity, daily routines, and mental well-being since transitioning to WFH per state restrictions following Covid-19. Sampling targeted a population of adults 18 years old or older, living in the Seattle region (King, Kitsap, Pierce, 
and Snohomish Counties). The survey provided no financial incentive for participation. Both surveys were distributed online through professional email lists, including public agencies, non-governmental organizations, community groups, universities and colleges. Protocols were approved by the University of Washington Internal Review Board.

Survey respondents came from $87 \%$ of the region ZIP codes. They totaled 4,151 with 3,402 responding to the spring survey and 749 to the fall. The present study included the 2,678 (65\%) who had shifted from working away to WFH since March 2020. Respondents from the two survey waves shared similar characteristics (Table 1). Compared to the region's population (US Census Bureau 2019), more respondents identified as female (69\% in spring and $64 \%$ in fall vs. $50 \%$ in the region), had a graduate degree ( $52 \%$ and $54 \%$ vs. $17 \%$ region), and belonged to households with incomes above $\$ 150,000$ (34\% and $38 \%$ vs. $27 \%$ region). There were fewer 18 -to-29-year-old adults (18\% and $13 \%$ vs. $22 \%$ region).

\section{Variables}

Analyses were conducted at the respondent level. The outcome variable was self-reported change in productivity since respondents started WFH. Three response options were provided: no change, a decrease, or an increase in productivity. Productivity was treated as an ordinal variable with decrease in productivity as the reference.

To explain changes in teleworking productivity over time, we used factors identified as significantly associated with teleworking productivity in our previous study (Shi et al. 2020). Factors were organized into four domains: socioeconomic status (SES), pre-pandemic commute trip mode and duration, lifestyle changes, and mental wellbeing (Table 1). All respondents reported commute duration for each mode (walking, biking, transit, SOV, and HOV), with four ordinal levels (0-do not use, 1- <30min, 2- 31-60min, 3- >1 hour). Depression was evaluated using two questions from the Patient Health Questionnaire (Kroenke, Spitzer, and Williams 2003): how often respondents felt depressed or had little interest in doing things on a 4-point Likert scale $(0=$ Not at all; 1 = Several days; 2 = More than half the days; 3 = Nearly every day). The depression measure was indexed by summing all scaled question results, with higher scores indicating higher levels of perceived depression (range $=0$ to 6). The measures have been tested for validity in previous studies (CohenCline, Turkheimer, and Duncan 2015).

Change over the time of the pandemic was captured by adding a dummy variable that identified respondents from the spring and fall survey waves.

\section{Statistical Analysis}

Ordered logit models served to assess factors associated with self-reported changes in productivity. We first tested univariate models for all hypothesized predictors, with survey wave dummy added to examine the effect of time. 
Table 1. Characteristics of survey respondents who shifted from working away to WFH: their SES, pre-pandemic commute trip mode and duration, mental wellbeing status, and lifestyle changes since Covid-19( $\mathrm{N}=2,678)$.

\begin{tabular}{|c|c|c|c|}
\hline Variables & $\begin{array}{c}\text { Spring 2020 } \\
(\mathrm{N}=2,174)\end{array}$ & $\begin{array}{l}\text { Fall } 2020 \\
(N=504)\end{array}$ & $\begin{array}{c}\text { Overall } \\
(N=2,678)\end{array}$ \\
\hline \multicolumn{4}{|c|}{ Outcome } \\
\hline \multicolumn{4}{|l|}{ Productivity } \\
\hline Less productive & $839(39 \%)$ & $150(30 \%)$ & $989(37 \%)$ \\
\hline No change in productivity & $817(38 \%)$ & $224(44 \%)$ & 1041 (39\%) \\
\hline More productive & $518(24 \%)$ & $130(26 \%)$ & $648(24 \%)$ \\
\hline \multicolumn{4}{|c|}{ Domain: SES } \\
\hline \multicolumn{4}{|l|}{ Age } \\
\hline 18 to 29 & $389(18 \%)$ & $65(13 \%)$ & $454(17 \%)$ \\
\hline 30 and above & $1782(82 \%)$ & 439 (87\%) & 2221 (83\%) \\
\hline Missing & $3(0.1 \%)$ & $0(0 \%)$ & $3(0.1 \%)$ \\
\hline \multicolumn{4}{|l|}{ Gender } \\
\hline Female & $1499(69 \%)$ & $322(64 \%)$ & $1821(68 \%)$ \\
\hline Male & $646(30 \%)$ & $176(35 \%)$ & $822(31 \%)$ \\
\hline Other & $29(1 \%)$ & $6(1 \%)$ & $35(1 \%)$ \\
\hline \multicolumn{4}{|l|}{ Employment } \\
\hline Professional/Business & $1753(81 \%)$ & $283(56 \%)$ & $2036(76 \%)$ \\
\hline Education/Research & $371(17 \%)$ & $139(28 \%)$ & $510(19 \%)$ \\
\hline Other & $50(2 \%)$ & $82(16 \%)$ & $132(5 \%)$ \\
\hline \multicolumn{4}{|l|}{ Educational attainment } \\
\hline High school/Associate degree & $251(12 \%)$ & $60(12 \%)$ & $311(12 \%)$ \\
\hline Bachelor degree & $777(36 \%)$ & $171(34 \%)$ & 948 (35\%) \\
\hline Graduate degree & $1129(52 \%)$ & $270(54 \%)$ & $1399(52 \%)$ \\
\hline Other & $17(1 \%)$ & $3(1 \%)$ & $20(1 \%)$ \\
\hline \multicolumn{4}{|l|}{ Household Income } \\
\hline Below $\$ 150,000$ & $1380(63 \%)$ & 297 (59\%) & $1677(63 \%)$ \\
\hline Above $\$ 150,000$ & 735 (34\%) & $194(38 \%)$ & 929 (35\%) \\
\hline Missing & $59(2.7 \%)$ & $13(2.6 \%)$ & $72(2.7 \%)$ \\
\hline \multicolumn{4}{|l|}{ Housing Tenure } \\
\hline Own & $1411(65 \%)$ & $348(69 \%)$ & $1759(66 \%)$ \\
\hline Rent & $715(33 \%)$ & $147(29 \%)$ & $862(32 \%)$ \\
\hline Other & $48(2.2 \%)$ & $9(1.8 \%)$ & $57(2.1 \%)$ \\
\hline \multicolumn{4}{|l|}{ Living arrangement } \\
\hline Partner (without children) & $876(40 \%)$ & $218(43 \%)$ & $1094(41 \%)$ \\
\hline Live alone & $316(15 \%)$ & $70(14 \%)$ & $386(14 \%)$ \\
\hline Roommates, friends, relatives & $308(14 \%)$ & $70(14 \%)$ & $378(14 \%)$ \\
\hline Children (with or without partner) & $619(28 \%)$ & $135(27 \%)$ & $754(28 \%)$ \\
\hline Other & $55(3 \%)$ & $11(2 \%)$ & $66(2 \%)$ \\
\hline \multicolumn{4}{|l|}{ Vehicle Ownership } \\
\hline Yes & 2029 (93\%) & $476(94 \%)$ & 2505 (94\%) \\
\hline No & $145(7 \%)$ & $28(6 \%)$ & $173(6 \%)$ \\
\hline \multicolumn{4}{|l|}{ Having a dog } \\
\hline Yes & 756 (35\%) & 171 (34\%) & 927 (35\%) \\
\hline No & 1418 (65\%) & $333(66 \%)$ & 1751 (65\%) \\
\hline
\end{tabular}

Sleep quality and quantity 
1-Decreased a lot

2-Decreased somewhat

3-No change

4-Increased somewhat

5-Increased a lot

Not Applicable

Amount of food consumed

1-Decreased a lot

2-Decreased somewhat

3-No change

4-Increased somewhat

5-Increased a lot

Not Applicable

Amount of Exercise

1-Decreased a lot

2-Decreased somewhat

3-No change

4-Increased somewhat

5-Increased a lot

Not Applicable

Time on social media

1-Decreased a lot

2-Decreased somewhat

3-No change

4-Increased somewhat

5-Increased a lot

Not Applicable

Time on personal hobby

1-Decreased a lot

2-Decreased somewhat

3-No change

4-Increased somewhat

5-Increased a lot

Not Applicable

$$
\begin{gathered}
176(8 \%) \\
531(24 \%) \\
725(33 \%) \\
610(28 \%) \\
132(6 \%) \\
0(0 \%) \\
21(1 \%) \\
258(12 \%) \\
1060(49 \%) \\
738(34 \%) \\
97(4 \%) \\
0(0 \%)
\end{gathered}
$$

567 (26\%)

$552(25 \%)$

297 (14\%)

542 (25\%)

$207(10 \%)$

$9(0 \%)$

$23(1 \%)$

$76(3 \%)$

$828(38 \%)$

781 (36\%)

$293(13 \%)$

$173(8 \%)$

$118(5 \%)$

$194(9 \%)$

$862(40 \%)$

701 (32\%)

$193(9 \%)$

106 (5\%)

$\begin{array}{cc}36(7 \%) & 212(8 \%) \\ 129(26 \%) & 660(25 \%) \\ 188(37 \%) & 913(34 \%) \\ 126(25 \%) & 736(27 \%) \\ 24(5 \%) & 156(6 \%) \\ 1(0 \%) & 1(0 \%) \\ & \\ 6(1 \%) & 27(1 \%) \\ 56(11 \%) & 314(12 \%) \\ 286(57 \%) & 1346(50 \%) \\ 142(28 \%) & 880(33 \%) \\ 14(3 \%) & 111(4 \%) \\ 0(0 \%) & 0(0 \%)\end{array}$

155 (31\%)

722 (27\%)

707 (26\%)

365 (14\%)

632 (24\%)

243 (9\%)

9 (0\%)

$35(1 \%)$

\begin{tabular}{|c|c|c|c|}
\hline \multicolumn{4}{|c|}{ Domain: pre-pandemic commute trip duration (one-way) by mode } \\
\hline \multicolumn{4}{|l|}{ Walking } \\
\hline 0-Do not use & $1603(74 \%)$ & $375(74 \%)$ & $1978(74 \%)$ \\
\hline $1-<30 \min$ & $414(19 \%)$ & $78(15 \%)$ & $492(18 \%)$ \\
\hline $2-31$ to $60 \mathrm{~min}$ & $123(6 \%)$ & $43(9 \%)$ & $166(6 \%)$ \\
\hline 3->1 hour & $34(2 \%)$ & $8(2 \%)$ & $42(2 \%)$ \\
\hline \multicolumn{4}{|l|}{ Biking } \\
\hline 0-Do not use & $1830(84 \%)$ & $399(79 \%)$ & $2220(83 \%)$ \\
\hline $1-<30 \mathrm{~min}$ & $195(9 \%)$ & $58(12 \%)$ & $253(9 \%)$ \\
\hline $2-31$ to $60 \mathrm{~min}$ & $122(6 \%)$ & $37(7 \%)$ & $159(6 \%)$ \\
\hline 3->1 hour & $27(1 \%)$ & $10(2 \%)$ & $37(1 \%)$ \\
\hline \multicolumn{4}{|l|}{ Transit } \\
\hline 0-Do not use & 1018 (47\%) & 220 (44\%) & $1238(46 \%)$ \\
\hline $1-<30 \mathrm{~min}$ & 391 (18\%) & $96(19 \%)$ & 487 (18\%) \\
\hline $2-31$ to $60 \mathrm{~min}$ & $593(27 \%)$ & $140(28 \%)$ & 733 (27\%) \\
\hline
\end{tabular}

106 (4\%)

$1003(37 \%)$

$963(36 \%)$

341 (13\%)

230 (9\%)

158 (6\%)

$262(10 \%)$

1068 (40\%)

$840(31 \%)$

229 (9\%)

$121(5 \%)$ 


\begin{tabular}{|c|c|c|c|}
\hline 3->1 hour & $172(8 \%)$ & $48(10 \%)$ & $220(8 \%)$ \\
\hline \multicolumn{4}{|c|}{ Single Occupancy Vehicle (SOV) } \\
\hline 0-Do not use & $946(44 \%)$ & 207 (41\%) & $1153(43 \%)$ \\
\hline $1-<30 \mathrm{~min}$ & $735(34 \%)$ & $184(37 \%)$ & $919(34 \%)$ \\
\hline $2-31$ to $60 \mathrm{~min}$ & $424(20 \%)$ & $98(19 \%)$ & $522(19 \%)$ \\
\hline 3->1 hour & $69(3 \%)$ & $15(3 \%)$ & $84(3 \%)$ \\
\hline \multicolumn{4}{|c|}{ High Occupancy Vehicle (HOV) } \\
\hline 0-Do not use & $1882(87 \%)$ & $444(88 \%)$ & $2326(87 \%)$ \\
\hline $1-<30 \mathrm{~min}$ & $152(7 \%)$ & $30(6 \%)$ & $182(7 \%)$ \\
\hline $2-31$ to $60 \mathrm{~min}$ & $118(5 \%)$ & $25(5 \%)$ & $143(5 \%)$ \\
\hline 3->1 hour & $22(1 \%)$ & $5(1 \%)$ & $27(1 \%)$ \\
\hline \multicolumn{4}{|c|}{ Domain: mental wellbeing } \\
\hline \multicolumn{4}{|l|}{ Depression measure } \\
\hline Mean (SD) & $1.45(1.48)$ & $1.42(1.48)$ & $1.44(1.48)$ \\
\hline Median [Min, Max] & $1.00[0,6.00]$ & $1.00[0,6.00]$ & $1.00[0,6.00]$ \\
\hline \multicolumn{4}{|l|}{ Anxiety measure } \\
\hline Mean (SD) & $4.02(4.14)$ & $3.96(4.17)$ & $4.01(4.15)$ \\
\hline Median [Min, Max] & $3.00[0,24.0]$ & $3.00[0,24.0]$ & $3.00[0,24.0]$ \\
\hline
\end{tabular}

A base model was estimated with all variables significant in the univariate models as well as the survey wave dummy. We then tested interaction terms between each factor and the survey wave dummy and only reported those that were significant at $\mathrm{p}<0.05$ level. We conducted brant tests on the proportional odds assumption and results showed the assumptions were not violated (Brant 1990).

\section{Findings}

In our sample, productivity appeared to increase over the course of the pandemic. More respondents reported experiencing no change in productivity in the fall than in the spring ( $44 \%$ vs. $38 \%$ ) or being more productive $(26 \%$ vs. $24 \%$ ) (Table 1). After controlling for SES, pre-pandemic commute trip duration, lifestyle changes, and mental wellbeing status, fall respondents were $48 \%$ more likely than spring respondents to report experiencing no change or an increase in productivity (Table 2 Model 1). This suggested that people have adapted to teleworking gradually and positively as the pandemic subsisted. 
Table 2. Association between reported productivity, SES, pre-pandemic commute trip mode and duration, lifestyle changes, and mental wellbeing ( $\mathrm{N}=2,287)$.

\begin{tabular}{|c|c|c|c|c|c|c|c|}
\hline \multirow[b]{2}{*}{ Domains } & \multirow[b]{2}{*}{ Predictors } & \multicolumn{2}{|c|}{ Model 1} & \multicolumn{2}{|c|}{ Model 2} & \multicolumn{2}{|c|}{ Model 3} \\
\hline & & $O R$ & $p$ & $O R$ & $p$ & $O R$ & $p$ \\
\hline \multirow[t]{2}{*}{ Temporal Effect } & Spring 2020 & \multicolumn{2}{|c|}{ Reference } & \multicolumn{2}{|c|}{ Reference } & \multicolumn{2}{|c|}{ Reference } \\
\hline & Fall 2020 & 1.48 & $<0.001$ & 1.16 & 0.266 & 1.62 & 0.002 \\
\hline \multirow[t]{26}{*}{ SES } & Age & & & & & & \\
\hline & -18 to 29 & 0.52 & $<0.001$ & 0.51 & $<0.001$ & 0.51 & $<0.001$ \\
\hline & - 30 and above & \multicolumn{2}{|c|}{ Reference } & \multicolumn{2}{|c|}{ Reference } & \multicolumn{2}{|c|}{ Reference } \\
\hline & Employment & & & & & & \\
\hline & - Professional/business & \multicolumn{2}{|c|}{ Reference } & \multicolumn{2}{|c|}{ Reference } & \multicolumn{2}{|c|}{ Reference } \\
\hline & - Education/research & 0.52 & $<0.001$ & 0.52 & $<0.001$ & 0.51 & $<0.001$ \\
\hline & - Others & 0.71 & 0.069 & 0.72 & 0.089 & 0.71 & 0.073 \\
\hline & Education & & & & & & \\
\hline & - High school/Associate degree & 1.41 & 0.012 & 1.42 & 0.011 & 1.43 & 0.010 \\
\hline & - Bachelor's degree & 1.34 & 0.001 & 1.36 & 0.001 & 1.34 & 0.001 \\
\hline & - Graduate and above & \multicolumn{2}{|c|}{ Reference } & \multicolumn{2}{|c|}{ Reference } & \multicolumn{2}{|c|}{ Reference } \\
\hline & - Other & 2.37 & 0.077 & 2.38 & 0.076 & 2.59 & 0.055 \\
\hline & Household income & & & & & & \\
\hline & - Below $\$ 150,000$ & \multicolumn{2}{|c|}{ Reference } & \multicolumn{2}{|c|}{ Reference } & \multicolumn{2}{|c|}{ Reference } \\
\hline & - Above $\$ 150,000$ & 1.16 & 0.102 & 1.03 & 0.791 & 1.16 & 0.100 \\
\hline & - Above $\$ 150,000 *$ Fall 2020 & & & 1.87 & 0.003 & & \\
\hline & Living arrangement & \multirow{2}{*}{\multicolumn{2}{|c|}{ Reference }} & \multirow{2}{*}{\multicolumn{2}{|c|}{ Reference }} & & \\
\hline & - Partner & & & & & \multicolumn{2}{|c|}{ Reference } \\
\hline & - Live alone & 0.96 & 0.725 & 0.97 & 0.789 & 1.00 & 0.981 \\
\hline & - Friends and relatives & 1.04 & 0.739 & 1.05 & 0.711 & 1.18 & 0.251 \\
\hline & - Children & 0.66 & $<0.001$ & 0.67 & $<0.001$ & 0.64 & $<0.001$ \\
\hline & - Other & 0.71 & 0.197 & 0.71 & 0.198 & 0.87 & 0.636 \\
\hline & - Live alone * Fall 2020 & & & & & 0.78 & 0.423 \\
\hline & - Friends and relatives * Fall 2020 & & & & & 0.52 & 0.045 \\
\hline & - Children *Fall 2020 & & & & & 1.21 & 0.439 \\
\hline & - Other * Fall 2020 & & & & & 0.31 & 0.094 \\
\hline \multirow[t]{2}{*}{ Lifestyle Changes } & Sleep quality and quantity & 1.10 & 0.019 & 1.10 & 0.019 & 1.10 & 0.017 \\
\hline & Time on social media & 0.86 & 0.002 & 0.86 & 0.002 & 0.85 & 0.002 \\
\hline
\end{tabular}




\begin{tabular}{|c|c|c|c|c|c|c|c|}
\hline & \multirow[b]{2}{*}{ Time on personal hobby } & \multicolumn{2}{|c|}{ Model 1} & \multicolumn{2}{|c|}{ Model 2} & \multicolumn{2}{|c|}{ Model 3} \\
\hline & & 1.10 & 0.024 & 1.10 & 0.028 & 1.10 & 0.024 \\
\hline \multirow[t]{2}{*}{ Pre-pandemic Commute Trip Duration } & Walking & 0.85 & 0.012 & 0.86 & 0.015 & 0.85 & 0.012 \\
\hline & Sov & 1.24 & $<0.001$ & 1.24 & $<0.001$ & 1.24 & $<0.001$ \\
\hline Mental Wellbeing & Depression measure & 0.82 & $<0.001$ & 0.82 & $<0.001$ & 0.83 & $<0.001$ \\
\hline
\end{tabular}

Note: Model 1- Base Model; Model 2- Household income*Fall 2020; Model 3-Living arrangement*Fall 2020. Grey cells: variables capturing temporal changes. 


\begin{tabular}{ccccc}
\hline & \multicolumn{2}{c}{ Below $\$ 150,000$} & \multicolumn{2}{c}{ Above $\$ 150,000$} \\
& OR & $p$ & OR & $p$ \\
\hline Fall 2020 - Spring 2020 & 1.16 & 0.27 & & $<.17$ \\
\hline${ }^{* * *} \mathrm{p}<0.001^{* *} \mathrm{p}<0.01^{*} \mathrm{p}<0.05$ & & & & \\
\hline
\end{tabular}

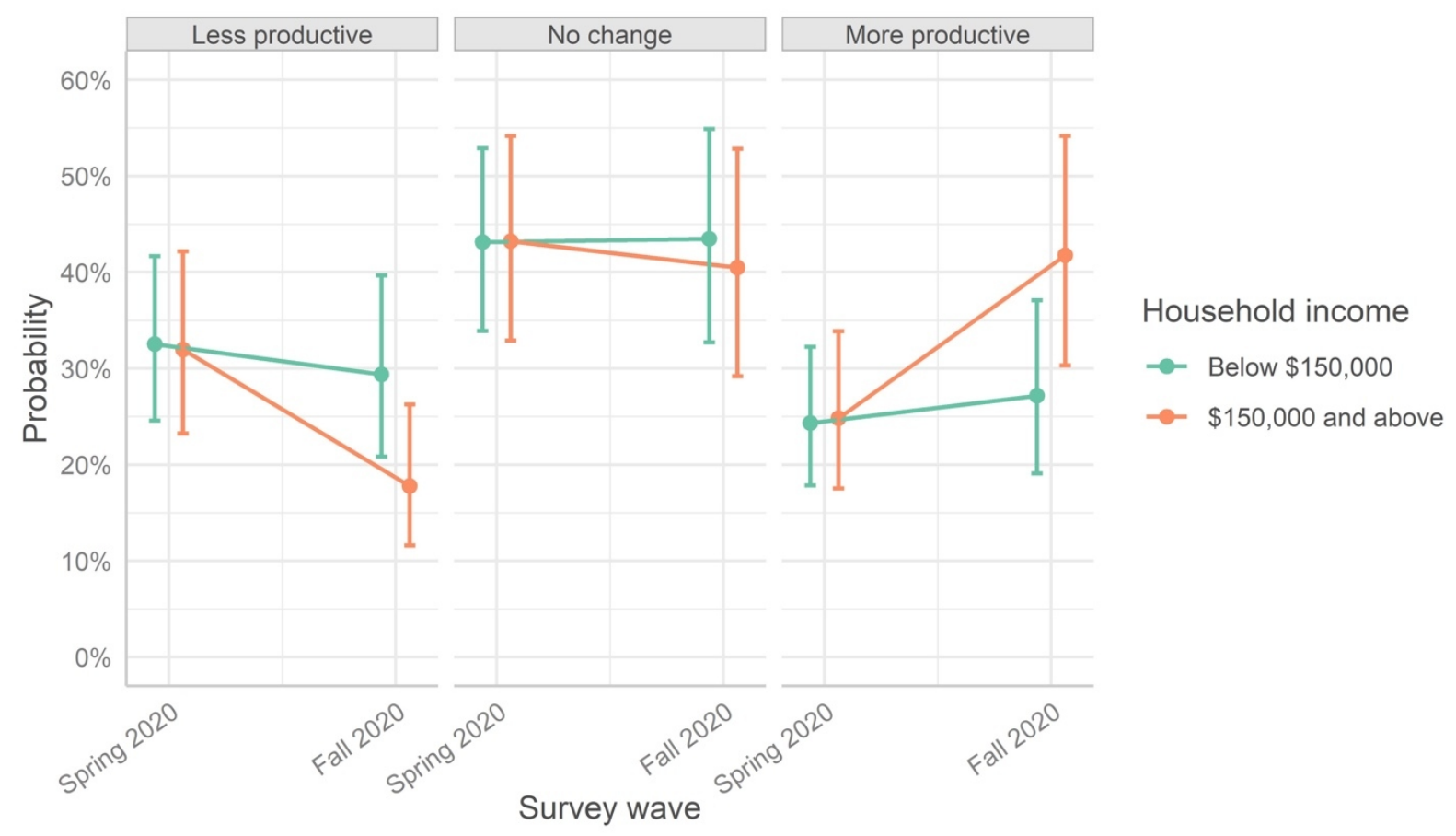

Figure 1. Modeled differences in the effect of income between the spring and fall survey.

Income and living arrangement had a moderating effect on the capacity to adapt to teleworking. Change in self-reported productivity was different for people coming from different household income brackets (Table 2 Model 2). A significantly higher proportion of people with household incomes above $\$ 150,000$ reported being more productive compared to spring respondents (Table 3, Figure 1). Therefore, people who were more financially advantaged appeared to be better able to adapt to teleworking than those with lower incomes. A likely explanation is that higher income translates into greater access to resources and the ability to make timely adjustments, such as enhancing internet infrastructure, having a spare room for work, having satisfactory child care, etc. (Cox et al. 2020; Prime, Wade, and Browne 2020), all conditions that may lead to greater productivity.

Living arrangement had a moderating effect on productivity change. Compared to other living arrangements, people living with roommates and friends experienced a decrease in productivity (Table 2 Model 3). However, the effect was no longer significant after considering that of income (Appendix 
Table A1 Model 4), likely because household income and living arrangement were correlated, where people living with friends and relatives tended to also have household incomes below $\$ 150,000$ (Appendix Table A2).

Other factors found to influence teleworking productivity in the spring survey (Shi et al. 2020) (age, pre-pandemic commuting patterns, lifestyle changes, and mental wellbeing status) remained significant after considering the temporal effect. The pooled data structure used did not infer causal relations, however, findings strongly suggested that many higher income workers could continue to WFH past the pandemic while remaining productive, and could potentially reduce the number of future commute trips. Extra effort should be made to support those with fewer resources to telework productively if they choose to.

\section{Acknowledgements}

This work has been funded in part by the US Department of Transportation's University Transportation Center program through the Pacific Northwest Regional University Transportation Center (PacTrans) and by the Puget Sound Regional Council (PSRC). Views expressed in the paper do not represent those of the sponsors and the authors are responsible for all errors that may exist. 


\section{REFERENCES}

Brant, Rollin. 1990. "Assessing Proportionality in the Proportional Odds Model for Ordinal Logistic Regression.” Biometrics, 1171-78.

Cohen-Cline, Hannah, Eric Turkheimer, and Glen E. Duncan. 2015. "Access to Green Space, Physical Activity and Mental Health: A Twin Study." J Epidemiol Community Health 69 (6): 523-29.

Cox, Natalie, Peter Ganong, Pascal Noel, Joseph Vavra, Arlene Wong, Diana Farrell, Fiona Greig, and Erica Deadman. 2020. "Initial Impacts of the Pandemic on Consumer Behavior: Evidence from Linked Income, Spending, and Savings Data.” Brookings Papers on Economic Activity 2020 (2): $35-82$.

Kazekami, Sachiko. 2020. "Mechanisms to Improve Labor Productivity by Performing Telework." Telecommunications Policy 44 (2): 101868.

Kroenke, Kurt, Robert L. Spitzer, and Janet BW Williams. 2003. "The Patient Health Questionnaire-2: Validity of a Two-Item Depression Screener.” Medical Care, 1284-92.

Nakrošienė, Audronė, Ilona Bučiūnienè, and Bernadeta Goštautaitè. 2019. "Working from Home: Characteristics and Outcomes of Telework." International Journal of Manpower.

Pigini, Claudia, and Stefano Staffolani. 2019. "Teleworkers in Italy: Who Are They? Do They Make More?” International Journal of Manpower.

Prime, Heather, Mark Wade, and Dillon T. Browne. 2020. "Risk and Resilience in Family Well-Being during the COVID-19 Pandemic.” American Psychologist 75 (5): 631-43. https://doi.org/10.1037/ amp0000660.

Shi, Xiao, Anne Vernez Moudon, Brian HY Lee, Qing Shen, and Xuegang Jeff Ban. 2020. "Factors Influencing Teleworking Productivity-a Natural Experiment during the COVID-19 Pandemic.” Findings.

Soares, Sergei, Florence Bonnet, and Janine Berg. 2021. "Working from Home during the COVID-19 Pandemic: Updating Global Estimates Using Household Survey Data.” VoxEU.Org (blog). April 25, 2021. https://voxeu.org/article/working-home-during-covid-19-pandemic-updated-estimates.

US Census Bureau. 2019. “2019 American Community Survey Single-Year Estimates.” Census.Gov. 2019. https://www.census.gov/newsroom/press-kits/2020/acs-1year.html. 


\section{Appendix}

Table A1.

Model 4

\begin{tabular}{|c|c|c|c|}
\hline Domains & Predictors & $O R$ & $p$ \\
\hline \multirow[t]{2}{*}{ Temporal Effect } & Spring 2020 & Reference & \\
\hline & Fall 2020 & 1.25 & 0.231 \\
\hline \multirow[t]{26}{*}{ SES } & Age & & \\
\hline & -18 to 29 & 0.51 & $<0.001$ \\
\hline & -30 and above & Reference & \\
\hline & Employment & & \\
\hline & - Professional/business & Reference & \\
\hline & - Education/research & 0.51 & $<0.001$ \\
\hline & - Others & 0.72 & 0.085 \\
\hline & Education & & \\
\hline & - High school/Associate degree & 1.43 & 0.009 \\
\hline & - Bachelor's degree & 1.36 & 0.001 \\
\hline & - Graduate and above & Reference & \\
\hline & - Other & 2.61 & 0.054 \\
\hline & Household income & & \\
\hline & - Below $\$ 150,000$ & Reference & \\
\hline & - Above $\$ 150,000$ & 1.05 & 0.663 \\
\hline & - Above $\$ 150,000 *$ Fall 2020 & 1.72 & 0.015 \\
\hline & Living arrangement & & \\
\hline & - Partner & Reference & \\
\hline & - Live alone & 0.97 & 0.825 \\
\hline & - Friends and relatives & 1.15 & 0.343 \\
\hline & - Children & 0.65 & $<0.001$ \\
\hline & - Other & 0.87 & 0.626 \\
\hline & - Live alone * Fall 2020 & 0.98 & 0.953 \\
\hline & - Friends and relatives * Fall 2020 & 0.62 & 0.152 \\
\hline & - Children * Fall 2020 & 1.17 & 0.513 \\
\hline & - Other * Fall 2020 & 0.31 & 0.098 \\
\hline \multirow[t]{3}{*}{ Lifestyle Changes } & Sleep quality and quantity & 1.10 & 0.018 \\
\hline & Time on social media & 0.85 & 0.001 \\
\hline & Time on personal hobby & 1.10 & 0.028 \\
\hline \multirow{2}{*}{$\begin{array}{l}\text { Pre-pandemic Commute } \\
\text { Trip Duration }\end{array}$} & Walking & 0.86 & 0.015 \\
\hline & SOV & 1.24 & $<0.001$ \\
\hline Mental Wellbeing & Depression measure & 0.82 & $<0.001$ \\
\hline
\end{tabular}

Note: Model 4 - Household income*Fall $2020+$ Living arrangement ${ }^{*}$ Fall 2020. 
Table A2. Distribution of Household Income by Living Arrangement.

\begin{tabular}{|c|ccccc|}
\hline $\begin{array}{c}\text { Living Arrangement } \\
\text { Household Income }\end{array}$ & partner & live alone & roommates, friends, relatives & children & other \\
\hline Below $\$ 150,000$ & $632(58 \%)$ & $350(91 \%)$ & $300(79 \%)$ & $354(47 \%)$ & $41(62 \%)$ \\
Above $\$ 150,000$ & $437(40 \%)$ & $29(8 \%)$ & $54(14 \%)$ & $387(51 \%)$ & $22(33 \%)$ \\
Missing & $25(2 \%)$ & $7(2 \%)$ & $24(6 \%)$ & $13(2 \%)$ & $3(5 \%)$ \\
\hline Correlation & & & $0.34(\mathrm{p}<0.001)$ & \\
\hline
\end{tabular}

